\title{
Constructing Application-specific Memory Hierarchies on FPGAs
}

\author{
Harald Devos $^{1}$, Jan Van Campenhout ${ }^{1}$, \\ Ingrid Verbauwhede ${ }^{2}$, and Dirk Stroobandt ${ }^{1}$ \\ 1 Parallel Information Systems, ELIS-Dept., Ghent University, \\ Sint-Pietersnieuwstraat 41, B-9000 Gent, Belgium \\ \{harald.devos I jan. vancampenhout/dirk.stroobandt\}@elis.UGent. be \\ ${ }_{2}$ Katholieke Universiteit Leuven, ESAT \\ Kasteelpark Arenberg 10, B-3001 Leuven-Heverlee, Belgium \\ Ingrid.Verbauwhede@esat.kuleuven. be
}

\begin{abstract}
The high performance potential of an FPGA is not fully exploited if a design suffers a memory bottleneck. Therefore, a memory hierarchy is needed to reuse data in on-chip buffer memories and minimize the number of accesses to off-chip memory. Buffer memories not only hide the external memory latency, but can also be used to remap data and augment the on-chip bandwidth through parallel access of multiple buffers. This paper discusses the differences and similarities of memory hierarchies on processor- and on FPGA-based systems and presents a step-by-step methodology to construct a memory hierarchy on an FPGA.
\end{abstract}

\section{Introduction}

FPGAs (Field Programmable Gate Arrays) offer a high computational power thanks to the massive parallelism available and the huge on-chip bandwidth. However, the total on-chip memory size is fairly small. Usually an off-chip memory is needed. The bandwidth to this external memory may become a bottleneck, especially for data-intensive applications, such as video coding and image processing. Typically, this memory is made in a technology, e.g., SDRAM, with an indeterministic latency and with a low bandwidth if transfers are not done in burst mode. This indeterministic latency may also be caused by the fact that the main memory is shared with other cores on the FPGA. To reduce the bandwidth requirements a memory hierarchy is needed. If frequently used data is stored in on-chip buffers, the off-chip memory accesses can be reduced and grouped into bursts.

Using multiple parallel accessible memory banks (Fig. 1(a)) increases the available off-chip memory bandwidth. On the other hand, using multiple onchip buffers increases the on-chip memory bandwidth (Fig. 1(b)). If the reuse of buffered data is high enough, i.e. the transfers between external memory and onchip buffers are within the bandwidth constraints, the latter solution is better since the accesses to on-chip memory are faster and more power efficient. If 


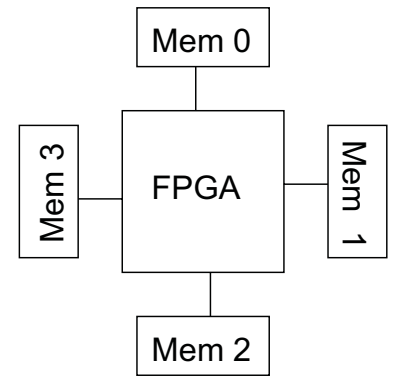

(a)

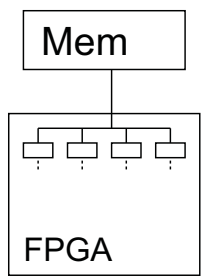

(b)

Fig. 1. Multiple memory banks (a). Single memory bank with multiple on-chip buffer memories (b).

the use of multiple external memory banks is unavoidable, a memory hierarchy for each bank should be considered and the memory access patterns should be optimized to use these hierarchies.

In processor-based systems a memory hierarchy consists of caches or scratchpad memories [1]. This is a fixed memory hierarchy and the application code has to be optimized to optimally use this given memory structure, e.g., by doing loop transformations to increase the data locality, i.e. to bring accesses to the same or neighboring data elements closer together in time $[2,3]$. On an FPGA the designer has to construct the memory hierarchy using the available memory blocks. This offers the freedom to build an application-specific memory hierarchy, i.e. to also adjust the memory system to the application and not only the application to the memory hierarchy. An in-depth comparison between memory systems on processors and on FPGAs is presented in Sect. 2.

This paper does not focus on ways to improve the locality of data accesses or map data to memories. Instead, we focus on hardware implementation aspects of building a memory hierarchy and the impact of choices made by the way data is mapped onto buffers. The influence of address expressions on circuit complexity is studied in Sect. 3, which offers many optimization opportunities.

A step-by-step methodology is given to insert a memory hierarchy into a system (Sect. 4). As a case study an implementation of an Inverse Discrete Wavelet Transform (IDWT) will be extended with a memory hierarchy and integrated in a video decoder on an FPGA (Sect. 5).

\section{Comparison of Memory Systems on Processors and on FPGAs}

The target of using memory hierarchies is the same for processors and for architectures built on FPGAs: storing frequently used data in buffer memories close to the functional units to minimize the data access times. In both cases 


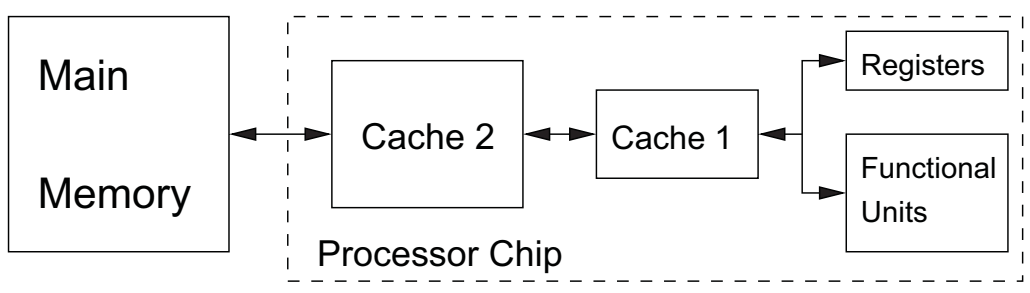

Fig. 2. Memory hierarchy on a typical processor

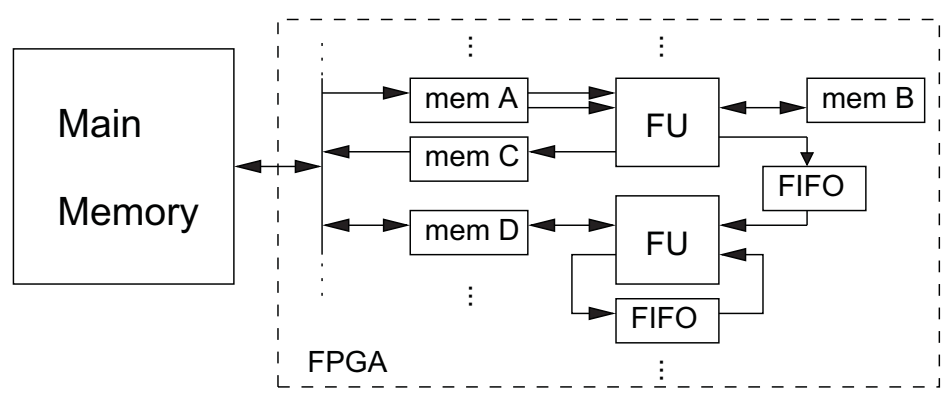

Fig. 3. Example memory hierarchy on an FPGA (FU = Functional Unit)

the application should be adjusted to benefit from such a memory system using techniques such as register promotion [4] or loop transformations [2,3] to improve the temporal and spatial locality. However, there are many differences caused by implementation aspects.

Processors have a fixed memory hierarchy consisting of a main memory, caches at several levels and one or more register files (Fig. 2). The sizes of all the memories are fixed and applications need to be adjusted to fit these sizes. Caches typically have a replacement policy so programmers should not care about explicit control of transfers between main memory and caches. A disadvantage is that the choice of data that is overwritten by newly fetched data is not always optimal for the given application. Scratch-pad memories may offer a better solution when the data accesses are known at compile time [1,5]. Prefetching [6] of data may hide external access times by overlapping them with computations. In cache-based systems a hash function is typically applied to the addresses which may cause irregular problem size / performance characteristics caused by (e.g. $64 \mathrm{~K})$ aliasing [7].

FPGAs have no standard memory hierarchy. They contain lots of memory blocks which have to be combined to build a custom memory system (e.g. Fig. 3). There are many similarities with scratch-pad memory based systems: user-defined explicit data transfers, burst transfers (cf. prefetch) between (external and internal) memories in parallel with computations, .... 


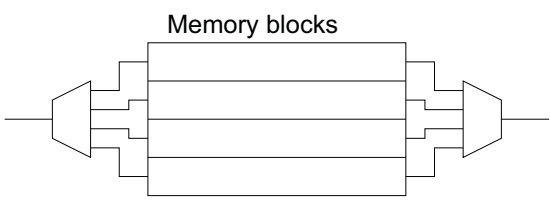

(a)

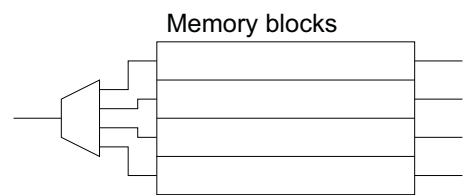

(b)

Fig. 4. Multiple memory blocks combined with (de)multiplexers to form larger buffers (a) can be accessed in parallel (b).

However, there are also lots of differences: processors typically have a vertical memory hierarchy while on an FPGA the memory system can be made more horizontally, i.e. placing several memories next to each other on a certain level such that they can be accessed in parallel by the functional units. FIFOs and local memories may be used, next to registers, to store intermediate results of functional units and pass data between them. As such there is a tremendous onchip bandwidth. If the intermediate data sets are too large, part of it has to be stored off-chip, passing through I/O buffers which aid in grouping the memory transfers into bursts.

Due to the limited size of memory blocks on FPGAs, larger buffer memories are constructed by combining multiple memory blocks (Fig. 4(a)). These memory blocks can also be accessed independently. This allows parallel accesses to the buffers which may reduce the execution time (number of clock cycles) (Fig. 4(b)). The usage of dual-port memories allows to transfer data between the main memory and the buffers in parallel with the operation of the functional unit, and to use different clock domains for both tasks. Also the fact that dual-port memories can have different word sizes at both ports can be exploited.

We can conclude that because of the similarities with scratch-pad based systems many of the optimizing transformations developed for processor-based systems also have to be applied for FPGA-based systems. For example, in the work of $\mathrm{Hu}$ et al. [3] the data sets used in each loop of a loop nest are determined and a data reuse analysis leads to the construction of a data reuse tree. Nodes of this tree representing data subsets (copy candidates) are then mapped to the scratch-pad memory according to the size constraints. This method could be extended to application-specific memory systems where different copy candidates may, e.g., be mapped to different memories with sizes fit to the size of the copy candidate (of course within resource constraints).

The additional benefits of custom-built memory systems need additional optimization techniques. Next to this, automation of the construction of a memory system is needed. A methodology for the construction of a memory hierarchy will be presented in Sect. 4 . 
Table 1. Influence of address expressions on circuit complexity for the Sobel Edge Detection on an Altera Stratix EP1S25F1020C5. LE = number of Logic elements, DSP $=$ number of DSP blocks (9-bit equivalent), Mem bits = size of on-chip memory. Cycles and execution time $(T)$ for an image of $320 \times 320$ pixels. A one cycle access time to external memory is assumed.

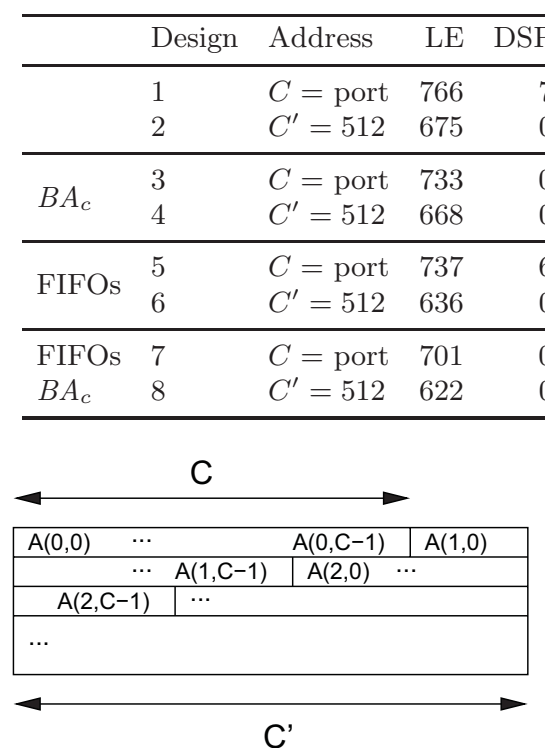

(a) $\operatorname{Address}(A(i, j))=i \times C+j$

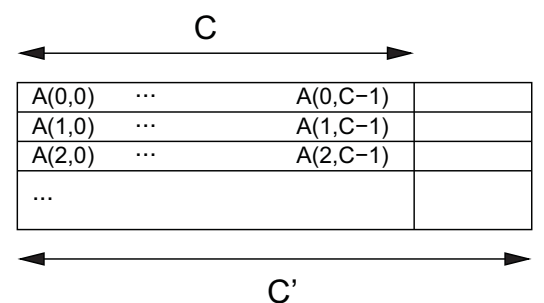

(b) $\operatorname{Address}(A(i, j))=i \times C^{\prime}+j$

Fig. 5. By inserting empty space into the memory, address expressions can be simplified.

\section{Influence of Address Complexity on Circuit Complexity}

The way memory addresses are computed has an influence on the circuit complexity of an implementation. Consider for example an image processing system that reads a 2 -dimensional array $A$. The typical way to store this array leads to address expressions with a multiplication:

$$
\operatorname{Address}(A(i, j))=B A+i \times C+j,
$$

with $B A$ the base address and $C$ the number of columns. If $C$ is not a power of 2 or $C$ is a parameter, which only receives a value during execution, this is an expensive operation. Without loss of generality we will further assume that $B A=0$ (Fig. 5(a)).

A straight-forward simplification is to align all lines to a multiple of a power of 2. This is shown in Fig. 5(b), where $C^{\prime}$ is the smallest power of 2 for which $C^{\prime} \geq C$, if $C$ is known, or $C^{\prime} \geq C_{\max }$ if $C$ is a parameter with $C_{\max }$ as the maximal possible value. This leads to an increase of the memory size with a factor 
$C^{\prime} / C$ but simplifies the address calculation complexity. ${ }^{3}$ Quantitative figures for an implementation of the Sobel Edge Detection algorithm can be found in Table 1. Since here the goal of the experiments is to study the influence of address expressions, no buffer memories are inserted yet and a one cycle access time to memory is assumed (as if the memory was on-chip). In design 1, DSP blocks are utilized to implement the multiplication which is eliminated by using $C^{\prime}$ in design 2 .

If an image is processed row by row (with some reuses of the lines above and below) the expensive multiplication can also be eliminated by incrementing the row base address with the number of columns at each row iteration:

$$
\operatorname{Address}(A(i, j))=\operatorname{Address}\left(A\left(i_{c}+i_{o s}, j\right)\right)=B A_{c}+i_{o s} \times C+j,
$$

where $i_{c}$ is the iterator pointing to the current row, and $B A_{c}$ is the address of the first element of this row $\left(B A_{c}=i_{c} \times C\right)$. Since $B A_{c}$ is augmented with $C$ every row iteration, no multiplication is needed. $i_{o s}$ is a small constant used to access the surrounding rows. In the example of the Sobel Edge Detection $i_{o s} \in$ $\{-1,0,1\}$ which does also not need a multiplier. As in the previous paragraph $C$ may be replaced with $C^{\prime}$. The corresponding designs are found in Table 1, designs 3 and 4 . Note that the designs using incremental address calculation (3 and 4) are faster than those not using it ( 1 and 2). However, this is not always the case. The designs 5-8 are similar to $1-4$, but use two FIFO buffers to store the two previously accessed image rows. This reduces the number of external memory accesses with a factor 3 and changes the influence of the address computations. Now, the fastest design (6) does not use incremental address calculation.

Accesses to on-chip memory have to be fast since they limit the speed of the functional unit. Therefore, the address expressions for these accesses have to be kept simple. Address calculations for off-chip memory accesses can be spread over several clock cycles, since only one evaluation is needed for an entire burst transfer. The methods discussed above to simplify addresses for off-chip memory are also applicable for on-chip memory. Since until now we assumed a one-cycle access time as if on-chip memory was used, we expect similar performance improvements for on-chip address calculation. A difference is that multiple addresses off-chip will be mapped to the same buffer memory location.

Only a few lines can be stored in a buffer. If this number of lines R' is also set to a power of 2 and the mapping of lines of the image to lines of the buffer is done in a circular way the address becomes

$$
\begin{aligned}
\operatorname{Address}(A(i, j)) & =\left(i \bmod R^{\prime}\right) \times C^{\prime}+j \\
& =i(r-1 \text { downto } 0) \& j, \quad \text { with } R^{\prime}=2^{r} .
\end{aligned}
$$

As a result, only the least significant bits of $i$ have to be generated. Note that the addition is in fact only a concatenation, denoted with " $\&$ ".

\footnotetext{
${ }^{3}$ Note that when $C$ is only known at execution time and $C_{\max }$ is a power of two (as is often the case) no extra memory is needed.
} 
Using $C^{\prime}$ instead of $C$ now sacrifices on-chip memory. In the introduction, the limited amount of on-chip memory was mentioned as the motivation for using buffer memories, At first sight sacrificing memory does not seem a good option. However, the scale differs with an order of magnitude. For a typical image processing application an entire image does not fit into the memory. The largest subset of an image that is worth storing on-chip is in the order of a few lines (e.g., corresponding to the data reuses of a sliding window operation), which can be stored easily in the FPGA RAM blocks. This leaves room to sacrifice some memory space as long as the reuse set (these few lines) can be stored. Saving

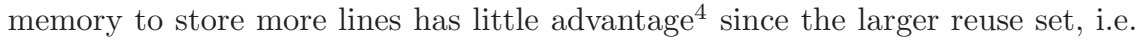
one image, will never fit on chip.

These methods differ from the address optimization techniques used when targeting processor-based systems (cf. Sect. 6). For processors, modulo operations have to be avoided to save instruction cycles. On FPGAs modulo operations by powers of two reduce the area and increase the maximal clock speed, which cannot be altered on processors.

\section{Step-by-step Construction of a Memory Hierarchy}

Many high-level synthesis tools generate one memory for each array in the input code. Also when building a design manually, it is easier to start the design process with multiple memories and only construct a memory hierarchy with one main memory later on. Therefore, we present a step-by-step design flow to transform a system with multiple memory banks, similar to the systems in Fig. 1(a) and $7(\mathrm{a})$, to a system with one external memory and on-chip buffers, similar to the systems in Fig. 1(b) and 7(c). Here, an overview of the flow is given. A detailed elaboration is found in the case study in Sect. 5 .

1. On-chip memories are added to contain intermediate data sets that are small enough to fit in them. This includes techniques such as register promotion. If results produced by one operation are consumed by another operation in the same order, FIFO buffers can be used.

2. The address expressions are optimized as described in Sect. 3. At this point synthesis is faster than after the entire memory system is constructed. Now, all addresses point to external memory but later on most of them will access on-chip buffers.

3. Buffers are inserted between each external memory and the functional unit.

The size is kept as large as the external memory itself so that no remapping of data and changes in address expressions are needed. In a later step the buffers will be reduced to actually fit on chip. A single copy transaction (for each memory/buffer pair) of all data to the buffer at the start of execution and a single transfer to the external memory at the end suffices for correct behavior. Separate I/O modules take care of these copy transactions. In the next step the transfers will be partitioned into smaller steps.

\footnotetext{
${ }^{4}$ Having the capacity to buffer more lines may be used, e.g., to enlarge the burst transfers or tolerate greater variance in the transactions to the external memory.
} 


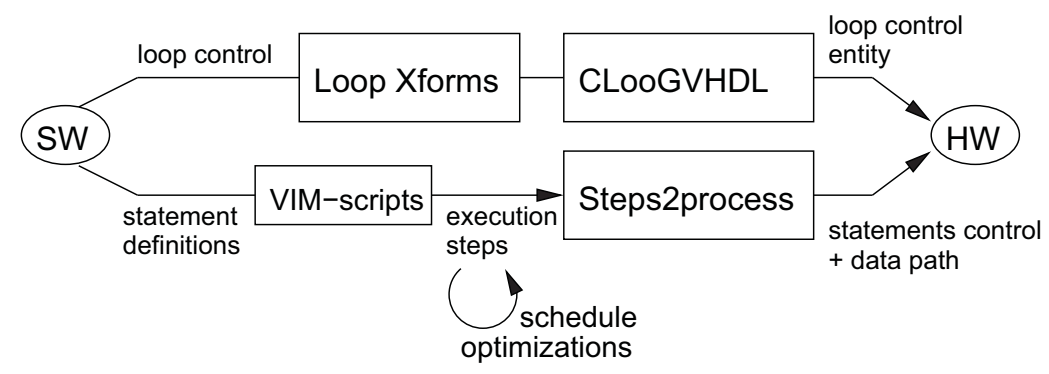

Fig. 6. Tool flow used to generate the IDWT implementation

4. The two large copy operations are split into smaller prefetch and store operations, such that at each moment only a small amount of data in the buffers is alive (= transferred and still needed). Synchronization between the data transfers and the operation of the functional unit ensures correct behavior. For this the technique of Hu et al. [3] to extract copy candidates can be used.

5. The data in the buffers is remapped such that the buffers can be resized and fit in on-chip memories. A hash function (cf. caches) translates the indices of the arrays into the new address expressions. The I/O modules take care of translations between on- and off-chip addresses, similar to the direct and strided mapping supported by the Impulse memory controller [8].

6. If desired, buffer memories may be split into smaller memories to allow parallel access (Fig. 4) and reduce the clock cycle count.

7. The external memories are merged to form one main memory. Base addresses are added to the addresses used in the prefetch and store transfers. The I/O modules are all connected to the same memory. Arbitration between the transfers is needed to avoid conflicts.

By doing the transformations in small steps, errors can be detected more easily and faster, since simulation is possible at any time. To increase the reusability, a modular architecture is used. When transferring the design to another platform only the I/O modules have to be adapted. By using hash functions instead of simply adapting the address expressions, the data mappings can be changed in an easier way, e.g., when a device upgrade offers the option to use more on-chip memory. This does not result in an area overhead since bits not used by the hash function will be optimized away by the synthesis tools (cf. (1)).

\section{Case Study: System Integration of an IDWT}

The 2-D Discrete Wavelet Transform (DWT) and its inverse (IDWT) are commonly used in image processing and compression applications, e.g., JPEG-2000. They contain operations commonly used in many other image processing applications, such as sliding window operations (2-D FIR filter), up- and downsampling, 


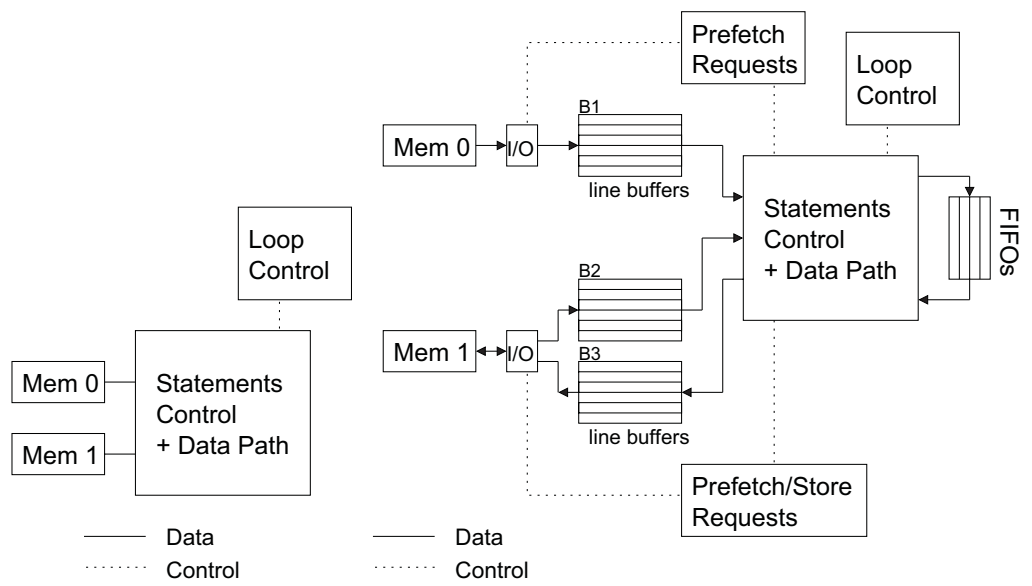

(a)

(b)

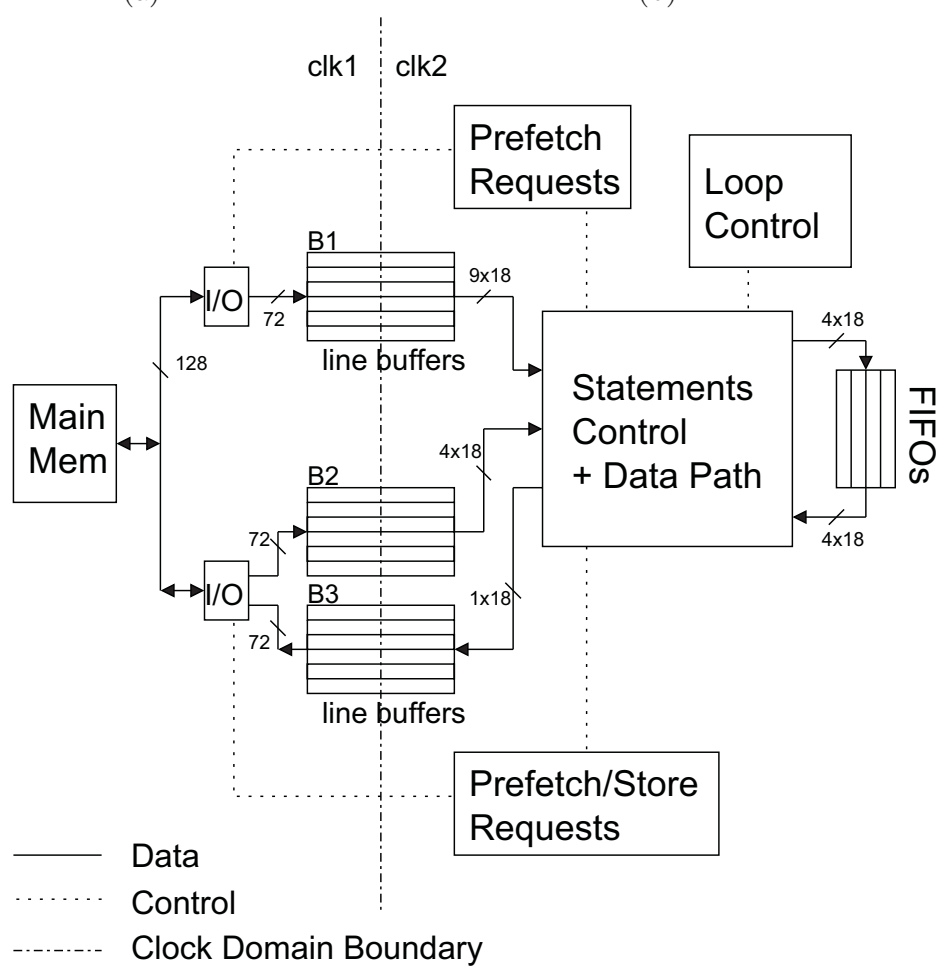

(c)

Fig. 7. Line-Based IDWT without memory hierarchy (a), after adding buffers (b), and after full system integration (c) 
mirroring at the borders, .... Therefore, results similar to those reported in this section are expected for many other image processing applications.

We will start from a design without memory hierarchy, but with a data locality optimized by loop transformations. It has been semi-automatically generated using the design flow shown in Fig. 6 as described in [9] and summarized in this paragraph. A software implementation in $\mathrm{C}$ is split into statement definitions and the loop control structure. Loop transformations are applied on the latter using the URUK/WRaP-IT tool suite [10] to improve the spatial locality, resulting in a so-called line-based variant. With CLooGVHDL a loop control entity is generated. The statement definitions are translated to a VHDL syntax using VIM-scripts [11]. ${ }^{5}$ The scripts translate array accesses into memory accesses with a one-cycle access time. The result is a list of execution steps for each statement. On this, schedule optimizations are done (also integrated into the scripts). The Steps2process tool generates a finite state machine to execute the statements based on the schedule specifications. The architecture of the generated design is shown in Fig. 7(a) and synthesis results are found in Table 2.

The fact that software code equivalent to the hardware is available can be exploited for the construction of a memory hierarchy as shown below.

\subsection{Adding New Hardware Structures}

The design tools mentioned above will be reused for the extension of the architecture with a memory system. New hardware constructs are inserted one after another, iterating over the following steps:

First, C preprocessor macros that simulate the behavior of the new construct are written. For example, a push and a pop macro to write to and read from a FIFO buffer. In $\mathrm{C}$ this is simulated by accessing an array and incrementing a counter. A VHDL block that corresponds with the new type of construct is written. Procedures or functions that correspond to the functionality of the $\mathrm{C}$ macros are written. For a FIFO this is the instantiation of FIFO entities and writing the VHDL procedures push_fifo and pop_fifo that access such a structure. Next, the VIM-scripts are extended to replace the $\mathrm{C}$ macros with the corresponding VHDL procedure or function calls. This work only has to be done for the first FIFO (or other new kind of block). Additional FIFOs reuse these constructs.

After the equivalence of the $\mathrm{C}$ and VHDL constructs and the mapping from the first to the second are tested, the new $\mathrm{C}$ macro can be used in all $\mathrm{C}$ code, where desired. Finally, the generated VHDL is tested and the impact of the new hardware structure may be examined after synthesis. Where possible optimizations are done. For example, removing unused data structures or doing schedule optimizations. After the introduction of the FIFOs more data elements can be accessed in parallel, which allows to shorten the schedules. These optimizations can be included in the scripts.

\footnotetext{
${ }^{5}$ This is only a temporary solution. Plans are to integrate a C parser. This is only an implementation issue and does not influence the methodology.
} 
This approach is similar to the way many high-level synthesis tools work. They extend $\mathrm{C}$ with macros that correspond to predefined hardware structures. Here, the difference is that new macros, not predefined by such tools, such as queues for prefetch requests, can be added and used with little effort.

\subsection{Inserting Buffers}

The extension of the design from Fig. 7(a) to (b) is done step-by-step following steps 1 through 6 listed in Sect. 4. For each new type of construct a flow as described in Sect. 5.1 is used.

1. Four FIFO buffers are inserted to transfer data from the vertical to the horizontal wavelet transformation. This halves the accesses to the main memories Mem 0 and Mem 1. Since the FIFO buffers can be accessed in parallel with the external memories the execution time is decreased (Table 2).

2. Since the dimensions of the input image are run-time parameters and $C_{\max }=$ 512 we align al image lines to a multiple of 512. By using a function call for the address calculation the address expression can easily be modified afterwards.

3. Mem 0 is only used for input values so applying step 3 of our methodology is straight-forward, i.e., one copy transaction at the start of execution to a buffer as large as Mem 0. Mem 1 is used for intermediate results. It passes the result of one level of the wavelet transform to the next. Therefore, copy operations are needed at the beginning and end of the execution of each wavelet transformation level.

4. The copy operations are split into transactions of one line of an image or wavelet sub-band. A block transfer system copies data from the main memories to and from the buffers. A queue of prefetch and store requests is kept in the Prefetch(/Store) Requests entities. A new fetch request is added a few row iterations before the data is needed. Synchronization points are used to ensure that when a line in one of the buffers is accessed, all transfers between that line and the main memory are finished. Therefore, if the system is not bandwidth limited, only in the beginning time is spent waiting for data. A block transfer is specified by the source and target address and the amount of data to be copied.

5. The buffers with sizes equal to the original memories are reduced to the line buffers B1(16 lines), B2(8 lines) and B3(8 lines). The address expressions referring to the buffer memories are extended with modulo expressions.

6. The line buffers B1 and B2 are split into parallel accessible buffers of one line to increase the on-chip bandwidth (cf. Fig. 4). This results in a large reduction in the number of clock cycles as shown in Table 2. The design in Fig. 7(b) is reached.

For the Stratix S60 the clock speed is increased after adding the memory hierarchy, thanks to the simplification of the address expressions. The clock speed on the S25 is lowered due to congestion in the FPGA routing (area usage of almost $70 \%$ ) but would be even worse without the address simplifications. 
Table 2. Synthesis results of the IDWT with and without memory hierarchy. CIF resolution $=352 \times 288$ pixels. $\mathrm{LE}=$ Logic Elements. Results obtained with Altera QuartusII v6.1 for the Altera Stratix EP1S25F1020C5 (S25C5) and EP1S60F1020C6 (S60C6, lower speed grade). The number of cycles and frame rate assume a one cycle $\left(1 / f_{\max }\right)$ access time to the main memories. With an SDRAM, this would be the performance when a cache running at $f_{\max }$ is used and no misses occur (i.e. the latency to the SDRAM is hidden, e.g., by prefetching). As a result this gives no measure of the performance improvement created by hiding the memory latency or reducing the number of off-chip accesses, but only of the improvement caused by the parallelism introduced by the memory system.

\begin{tabular}{|c|c|c|c|c|c|c|c|c|}
\hline \multirow{2}{*}{$\begin{array}{l}\text { Archi- } \\
\text { tecture }\end{array}$} & \multirow[t]{2}{*}{$\mathrm{LE}$} & \multirow{2}{*}{$\begin{array}{r}\text { Buf Mem } \\
\text { (bit) }\end{array}$} & \multirow{2}{*}{$\begin{array}{l}\text { DSP bl. } \\
(\# \text { Mul })\end{array}$} & \multirow{2}{*}{$\begin{array}{r}\text { Cycles } \\
(88 \times 72)\end{array}$} & \multicolumn{2}{|c|}{$f_{\max }(\mathrm{MHz})$} & \multicolumn{2}{|c|}{ Frames/s (CIF) } \\
\hline & & & & & $\mathrm{S} 25 \mathrm{C} 5$ & S60C6 & $\mathrm{S} 25 \mathrm{C} 5$ & S60C6 \\
\hline Fig. 7(a) & 10836 & 0 & $18(9)$ & 161037 & 50.12 & 43.40 & 19.71 & 17.07 \\
\hline$"+$ FIFOs & 10881 & 18432 & $18(9)$ & 148941 & 51.81 & 46.68 & 22.03 & 19.85 \\
\hline Fig. 7(b) & 17350 & 297504 & $18(9)$ & 59240 & 47.22 & 45.60 & 50.50 & 48.77 \\
\hline
\end{tabular}

\subsection{Further Integration}

Further integration work is needed to put the design on an Altera PCI Development Board with a Stratix EP1S60F1020C6 FPGA and $256 \mathrm{MiB}$ of DDR SDRAM memory (from Fig. 7(b) to (c)).

The content of the two main memories is mapped onto the single DDR SDRAM memory (step 7). An Avalon switch fabric [12] connects the DDR core (memory controller) with the I/O blocks. These blocks take care of the conversion of local addresses, used within the IDWT, to addresses in the global memory space. Since the 18 bit word width, used until now, does not correspond to the 128 bit data ports of the DDR controller, the word size at the left side of the line buffers is set to $4 \times 18=72$ bit and converted to and from $4 \times 32=128$ bit using sign extension and truncation.

The Avalon fabric only supports burst transfers that are a multiple of $16 \mathrm{~B}$ (128 bit) long and start at an address that is a multiple of $16 \mathrm{~B}$. Therefore, the lines in all wavelet sub-bands are aligned to a multiple of 128 bit in the main memory by letting each row start at a multiple of 512 pixels $(1$ pixel $=4 \mathrm{~B})$. This inserts more unused space than strictly needed, but memory space was not a problem in the DDR-memory and it simplifies address calculations, similar to the example in Fig. 5. A DMA controller (Direct Memory Access) drives the burst transfers [12].

To allow the memory controller and the wavelet transform to run at their maximal frequency, different clock domains are introduced. The dual-port memories offer a safe clock domain crossing for the data. For the control signals extra registers are inserted (brute-force synchronization).

Finally, other blocks are connected to the switch fabric to build the RESUME scalable wavelet-based video decoder described in [13]. It can decode 26.15 frames/s (clocking the DDR at $65 \mathrm{MHz}$, limited by the FPGA synthesis 
process). The IDWT on its own, clocked at $54 \mathrm{MHz}$ (reached with other tool settings than for Table 2), can transform 53 frames/s.

Power and energy dissipation figures of the IDWT implementation described above and a fully manual design without locality optimizations are found in [14]. The reduction of off-chip memory accesses leads to a large reduction of the dissipated energy which compensates the increased on-chip dissipation due to the larger amount of logic (total energy saving of a factor 2.2).

The final circuit has become much larger than the original one (Table 2). A large part of this circuitry is used for multiplexers connecting the parallel buffers of one line with the parallel computational units. In fact these lines are accessed in a circular way and this could in principle be implemented using FIFOs, as was done for the Sobel Edge Detection (Table 1, designs 5-8). This would remove the multiplexers and many address calculations. In fact these FIFOs should have been introduced in step 1 . The reason that this has not been done is because of the fact that there is an irregular behavior at the borders of each image or wavelet sub-band. There, mirroring is used to virtually extend the domain outside the borders which introduces an irregular memory access pattern. This hinders the use of FIFOs. The original program can be adapted to allow the use of FIFOs at these locations but this falls outside the scope of this paper and is left as future work.

\section{Related Work}

\subsection{Address Expressions}

A good data locality is needed to reuse data stored on-chip as much as possible. As mentioned above, loop transformations can improve this locality $[15,2,3]$.

A side effect of loop transformations is that address expressions may become complex. Therefore, address optimization techniques have been developed. Many exploit the repetitive evaluation of these expressions in a loop and use differences of the terms of an expression to calculate the next value, similar to the usage of $B A_{c}$ in Sect. 3 (Method of Difference). Sheldon et al. [16] present techniques to eliminate division and modulo operations, by inserting conditionals and using algebraic axioms and loop transformations. Most techniques optimize the evaluation of a given set of address expressions, possibly sharing logic among different address expressions [17]. Only a few remap data to simplify the address expressions $[18,8]$. Most methods are useful for both software and hardware implementations. A difference is that multiplications or modulo reductions with powers of 2 require shift operations on a processor but have no cost on an FPGA. Zissulescu et al. [19] focus on the generation of fast address calculation circuitry for FPGAs. They use number theory axioms and the method of difference to reduce the strength of the expressions but do not remap data to simplify them. 


\subsection{High-level Synthesis Tools}

In recent years many high-level synthesis (HLS) tools have been developed. Many of them extend a software programming language such as $\mathrm{C}$ with pragmas and macros to describe parallelism and the use of library constructs such as FIFOs.

Synfora PICO [20] does register promotion and tiling, but in order to further optimize the memory system the user has to insert FIFO constructs and pragmas to declare local memory.

NEC CyberWorkBench [21] has a user directive to specify if a 2-D array will be implemented with minimal size or with rows aligned to a power of two (cf. Sect. 3). The choice between the two is not made automatically.

Since sliding window operations are often used in image processing applications, several HLS tools focus on extracting such operations and mapping them on predefined architecture templates, e.g, SA-C [22] and ROCCC [23].

The Compaan/LAURA system converts an application into a Kahn-process network by automatically inserting FIFO buffers [24]. This could be used in the first step of our methodology. When a data set is not consumed in the order it is produced, reorder buffers are introduced. In the worst case these buffers may become too large to fit on chip. Loop transformations may be needed to avoid this.

A more extensive comparison of high-level synthesis tools with a focus on memory systems is found in Chapter 2 of [25].

We conclude that current HLS tools leave the design of the memory system to the designer but ease the construction of it by providing constructs to describe them at a higher level, or use predefined solutions for specific frequently used structures.

A common problem with HLS tools is the integration with user-built structures. This is done through communication by predefined (bus-)protocols which do not allow a tight integration or else the interaction should be described at a low level which removes the advantage of using HLS languages. An ideal highlevel synthesis tool should offer tight integration with user-built blocks by allowing to extend the set of macros or pragmas such that references to custom blocks can be recognized and dealt with on a footing of equality with the library blocks provided by the tools. This was the reason of using VIM-scripts. They allow to extend the set of macros to tightly integrate new blocks, such as the prefetch queue. The disadvantage was that some extra work is needed which we will try to eliminate by extending our tool suite.

\section{Conclusions and Future Work}

Application-specific memory hierarchies offer advantages that general purpose cache systems or scratch-pad memories do not offer, such as simplification of address calculation hardware and the increase of parallelism through buffer partitioning.

This paper presented a methodology to transform a system using multiple memories step-by-step into a system with a memory hierarchy connected to 
an external memory with unpredictable access times. Using a modular design description increases the reusability. With a good choice of data mapping in the buffers, addresses can be simplified to optimize the performance, possibly at the cost of a higher memory usage.

For many of the code transformation steps that were done partially manually, techniques to fully automate them are already available. Future work consists of integrating them and extend them where needed in the context of FPGA-based design. Better synthesis results are expected if the original code is transformed towards the targeted memory system, e.g., to increase the number of FIFO buffers.

\section{Acknowledgements}

This research is supported by the I.W.T. grant 060068 and the F.W.O. grant G.0475.05. Ghent University is a member of the HiPEAC Network of Excellence.

\section{References}

1. Banakar, R., Steinke, S., Lee, B.S., Balakrishnan, M., Marwedel, P.: Scratchpad memory: design alternative for cache on-chip memory in embedded systems. In: CODES '02: Proceedings of the tenth international symposium on Hardware/software codesign, New York, NY, USA, ACM Press (2002) 73-78

2. McKinley, K.S., Carr, S., Tseng, C.W.: Improving data locality with loop transformations. ACM Transactions on Programming Languages and Systems 18(4) (July 1996) 424-453

3. Hu, Q., Kjeldsberg, P.G., Vandecappelle, A., Palkovic, M., Catthoor, F.: Incremental hierarchical memory size estimation for steering of loop transformations. ACM Transactions on Design Automation of Electronic Systems 12(4) (2007) 50

4. Carribault, P., Cohen, A.: Applications of storage mapping optimization to register promotion. In: ICS '04: Proceedings of the 18th annual International Conference on Supercomputing, ACM Press (2004) 247-256

5. Geelen, B., Brockmeyer, E., Durinck, B., Lafruit, G., Lauwereins, R.: Alleviating memory bottlenecks by software-controlled data transfers in a data-parallel wavelet transform on a multicore DSP. In: Proceedings 1st Annual IEEE BENELUX/DSP Valley Signal Processing Symposium - SPS-DARTS. (April 2005) 143-146

6. Vanderwiel, S.P., Lilja, D.J.: Data prefetch mechanisms. ACM Comput. Surv. 32(2) (June 2000) 174-199

7. Shahbahrami, A., Juurlink, B., Vassiliadis, S.: Improving the memory behavior of vertical filtering in the discrete wavelet transform. In: CF '06: Proceedings of the 3rd conference on Computing Frontiers, New York, NY, USA, ACM Press (May 2006) 253-260

8. Zhang, L., Fang, Z., Parker, M., Mathew, Binu K.and Schaelicke, L., Carter, John B.and Hsieh, W.C., McKee, S.A.: The Impulse memory controller. IEEE Transactions on Computers 50(11) (November 2001) 1117-1132

9. Devos, H., Beyls, K., Christiaens, M., Van Campenhout, J., D'Hollander, E.H., Stroobandt, D.: Finding and applying loop transformations for generating optimized FPGA implementations. Transactions on High Performance Embedded Architectures and Compilers 1(2), LNCS 4050 (2007) 159-178 
10. Girbal, S., Vasilache, N., Bastoul, C., Cohen, A., Parello, D., Sigler, M., Temam, O.: Semi-automatic composition of loop transformations for deep parallelism and memory hierarchies. Int. J. Parallel Program. 34(3) (2006) 261-317

11. Oualline, S.: Vi IMproved (VIM). New Riders (2001)

12. Eeckhaut, H., Christiaens, M., Stroobandt, D.: Improving external memory access for Avalon systems on programmable chips. In: FPL'07, 17th International Conference on Field Programmable Logic and Applications. (August 2007)

13. Eeckhaut, H., Devos, H., Lambert, P., De Schrijver, D., Van Lancker, W., Nollet, V., Avasare, P., Clerckx, T., Verdicchio, F., Christiaens, M., Schelkens, P., Van de Walle, R., Stroobandt, D.: Scalable, wavelet-based video: from server to hardwareaccelerated client. IEEE Transactions on Multimedia 9(7) (November 2007) 15081519

14. Devos, H., Hendrik, E., Christiaens, M., Stroobandt, D.: Energy scalability and the RESUME scalable video codec. In Benini, L., Chang, N., Kremer, U., Probst, C.W., eds.: Power-aware Computing Systems. Number 07041 in Dagstuhl Seminar Proceedings, Dagstuhl, Germany, Internationales Begegnungs- und Forschungszentrum für Informatik (IBFI), Schloss Dagstuhl, Germany (January 2007) http: //drops.dagstuhl.de/opus/volltexte/2007/1112.

15. Wolf, M.E., Lam, M.S.: A data locality optimizing algorithm. In: PLDI '91: Proceedings of the ACM SIGPLAN 1991 conference on Programming language design and implementation, New York, NY, USA, ACM Press (June 1991) 30-44

16. Sheldon, J., Lee, W., Greenwald, B., Amarasinghe, S.: Strength reduction of integer division and modulo operations. In: Languages and Compilers for Parallel Computing: 14th International Workshop, LCPC. Volume 2624 of Lecture Notes in Computer Science., Cumberland Falls, Kentucky (August 2001) 1-14

17. Miranda, M.A., Catthoor, F.V., Janssen, M., De Man, H.J.: High-level address optimization and synthesis techniques for data-transfer-intensive applications. IEEE Transactions on Very Large Scale Integration (VLSI) Systems 6(4) (December 1998) 677-686

18. Panda, P.R., Catthoor, F., Dutt, N.D., Danckaert, K., Brockmeyer, E., Kulkarni, C., Vandecappelle, A., Kjeldsberg, P.G.: Data and memory optimization techniques for embedded systems. ACM Transactions on Design Automation of Electronic Systems 6(2) (2001) 149-206

19. Zissulescu, C., Kienhuis, B., Deprettere, E.: Expression synthesis in process networks generated by LAURA. In: 16th IEEE International Conference on Application-specific Systems, Architectures and Processors (ASAP). (July 2005) $15-21$

20. Synfora: PICO technology white paper (v.10)

21. NEC: CyberWorkBench. http://www.necst.co.jp/product/cwb/english/

22. Bohm, W., Hammes, J., Draper, B., Chawathe, M., Ross, C., Rinker, R., Najjar, W.: Mapping a single assignment programming language to reconfigurable systems. Journal of Supercomputing 21(2) (February 2002) 117-130

23. Guo, Z., Buyukkurt, B., Najjar, W., Vissers, K.: Optimized generation of datapath from $\mathrm{C}$ codes for FPGAs. In: DATE '05: Proceedings of the conference on Design, Automation and Test in Europe, Washington, DC, USA, IEEE Computer Society (2005) 112-117

24. Verdoolaege, S., Nikolov, H.N., Stefanov, T.P.: Improved derivation of process networks. In: ODES-4: 4th Workshop on Optimizations for DSP and Embedded Systems. (March 2006)

25. Devos, H.: Loop Transformations for the Optimized Generation of Reconfigurable Hardware. PhD thesis, Ghent University (February 2008) 